\title{
INTERMOLECULAR ENERGY TRANSFER BETWEEN LANTHANIDE COMPLEX IONS IN MICELLAR SOLUTION
}

\author{
W. Dong and C.D. Flint \\ Laser Laboratory, Department of Chemistry, Birkbeck College, University of London \\ Gordon House, 29 Gordon Square, London WC1H OPP, United Kingdom
}

Two different procedures for the study of the quenching of excited states by lanthanide complex ions on the surface of micelles are considered. In the first, excited $\mathrm{Tb}(\mathrm{pdc})_{3}^{3-}$ (pdc = pyridine 2,6-dicarboxylate) complex ions are held in close proximity to other $\operatorname{Ln}(\mathrm{pdc})_{3}^{3-}$ ions on the surface of cetyl trimethyl ammonium micelles. Efficient energy transfer is observed by the electric dipole-electric dipole mechanism with a mean interaction distance only slightly greater than that which exists in the crystalline complexes. In the second, the excited state of $\mathrm{Tb}(\mathrm{pdc})_{3}^{3-}$ is depopulated by energy transfer to the coexcited lowest excited triplet state of phenanthrene which is thereby excited to the second triplet state.

PACS numbers: 33.50.-j, 34.20.-b, 76.30.Kg

\section{Introduction}

In previous papers $[1-3]$ we have shown that $\operatorname{Ln}(\mathrm{pdc})_{3}^{3-}$ ions $(\mathrm{pdc}=$ pyridine 2,6-dicarboxylate) in dilute aqueous CTA (CTA = cetyl trimethyl ammonium) micellar solution strongly associate with the micellar surface. About 20 anions may surround a single cationic micelle. Whilst the interaction between the complex ions and the micelle is not sufficiently strong for the excited state energy levels to be significantly affected, the radiative and non-radiative relation rates for the excited state relaxations are markedly different for the cations associated with the anions associated with the micellar surface and the cations in free solution. Three factors contribute to this difference. Firstly, the exclusion of water from the outer coordination sphere of the complex ion by the presence of the quaternary nitrogen micellar "head" reduces the rate of excited state non-radiative relaxation. For $\mathrm{Tb}(\mathrm{pdc})_{3}^{3-}$ this effect is relatively small, since the non-radiative relaxation of the ${ }^{5} D_{4}$ state makes only a minor contribution to the decay of the excited state due to the large number of $\mathrm{O}-\mathrm{H}$ vibrational quanta required to bridge the energy gap between the lowest excited state and the ground state manifold. It is much more important for $\operatorname{Eu}(\mathrm{pdc})_{3}^{3-}$. Secondly, the distortion of the $\operatorname{Ln}(\mathrm{pdc})_{3}^{3-}$ complex 
from its idealised geometry, by the electrostatic effect of the head groups results in an increase in the transition dipole and hence of the radiative relaxation rate. Thirdly, the electrostatic and steric effects of the neighbouring anions on the micellar surface causes further distortion. Note that this effect will be additive to the effect of the head group, but the two can be partly distinguished by experiments by comparing the effect of mono-negative anions (such as $\mathrm{Br}^{-}$) and trinegative anions such as $\mathrm{Y}(\mathrm{pdc})_{3}^{3-}$. If the electrostatic interaction between two $\operatorname{Ln}(\mathrm{pdc})_{3}^{3-}$ ions on the micellar surface was sufficiently strong to cause a change in the transition dipoles, it seemed likely that they would be in sufficiently close proximity for energy transfer to occur between them. The first part of this paper deals with the observation of this transfer and the establishment of its mechanism. In the absence of micelles or other charged surfaces, energy transfer is barely detectable.

In addition, providing charged surfaces which may be utilised for energy transfer, micellar systems also provide hydrophobic interiors where neutral molecules may be localised. Two situations now arise. Firstly, it may be possible to hold two or more molecules within this small hydrocarbon-like region. The mean distance between such molecules will necessarily be small so that energy transfer between them may be significant [4]. Secondly, the mean distance between a molecule in the interior of a micelle and an ion or cation on the surface of a micelle will be small so that energy transfer from the interior to the surface or from the surface to the interior may be possible. All three situations have been observed.

\section{Experimental}

All experimental data in this paper are obtained in the same methods described in previous papers $[4,5]$.

\section{Theory}

In the rapid-diffusion limit (where all donors are effectively equivalent), the transfer rate, $k_{\mathrm{T}}$, is calculated by averaging over the space available to the donor

$$
k_{\mathrm{T}}=\iiint_{V} k_{0} \rho_{\mathrm{A}}\left(\frac{r}{R_{0}}\right)^{-6} \mathrm{~d} V,
$$

where $r$ is the variable distance between the donor and acceptor, $\rho_{\mathrm{A}}$ is the density of acceptor, $k_{0}$ is the decay constant of the excited state in the absence of acceptor, and $R_{0}$ is the critical distance for $50 \%$ energy transfer. For a simple homogeneous solution Eq. (1) simplifies to

$$
k_{\mathrm{T}}=1.33 \pi \rho_{\mathrm{A}} k_{0} R_{0}^{6} r_{\mathrm{a}}^{-3},
$$

where $r_{\mathrm{a}}$ is the distance of closest approach between the centre of the donor and acceptor [6]. For a microheterogeneous system such as a micellar solution, however, Eq. (2) is not valid.

For energy transfer on a surface of a spherical micelle with radius $R$ (see Fig. 1) Eq. (1) can be written as

$$
k_{\mathrm{T}}=\iint_{S} k_{0} \rho_{\mathrm{A}}\left(\frac{r}{R_{0}}\right)^{-6} \mathrm{~d} S
$$




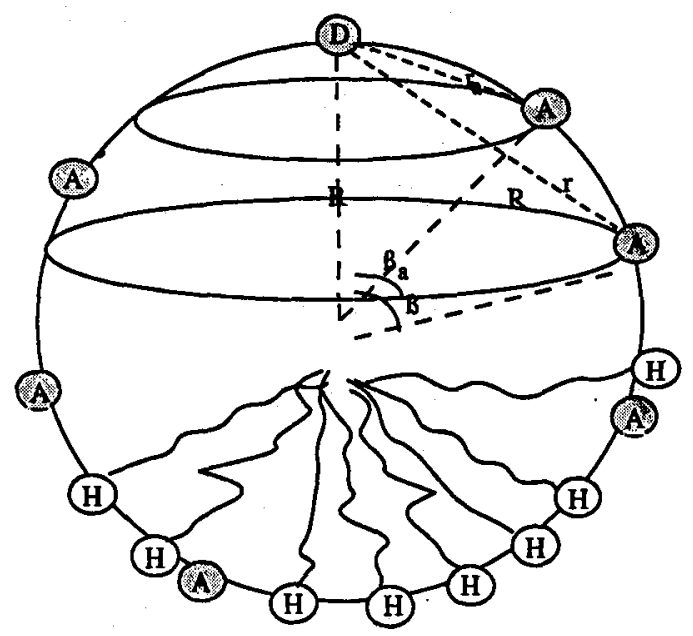

Fig. 1. Definition of angles for energy transfer on the surface of a micelle. $H$ indicates the surfactant head groups, A are the sites occupied by the donor and acceptor groups.

$S$ is the area available for donor-acceptor energy transfer and is given by

$$
S=2 \pi R^{2}(1-\cos \beta)
$$

where $\beta_{\mathrm{a}}$ is the angle corresponding to the distance of closest approach of a donor and an acceptor. The distance, $r$, between a donor and an acceptor on the micellar surface is given by

$$
\begin{aligned}
& r=2 R \sin (\beta / 2), \\
& r^{2}=4 R^{2} \sin 2^{2}(\beta / 2)=2 R^{2}(1-\cos \beta) .
\end{aligned}
$$

Equation (3) then can be expressed as follows by using Eq. (4) and Eq. (6):

$$
k_{\mathrm{T}}=\frac{1}{4} \pi k_{0} \rho_{\mathrm{A}} R^{-4} R_{0}^{6} \int_{\beta_{\mathrm{a}}}^{\pi} \frac{\sin \beta}{8 \sin ^{6} \frac{\beta}{2}} \mathrm{~d} \beta .
$$

After integration the rate constant for the dipole-dipole energy transfer on a micellar surface is

$$
k_{\mathrm{T}}=\frac{1}{32} \pi k_{0} \rho_{\mathrm{A}} R^{-4} R_{0}^{6}\left[\left(\sin ^{4} \frac{\beta_{\mathrm{a}}}{2}\right)^{-1}-1\right]
$$

or

$$
k_{\mathrm{T}}=(\pi / 2) \rho_{\mathrm{A}} k_{0} R_{0}^{6} r_{\mathrm{a}}^{-4} .
$$

Thus, the energy transfer rate, $k_{\mathrm{T}}$, on the micellar surface in two dimensions is proportional to $r_{\mathrm{a}}^{-4}$.

In the presence of the acceptor the lifetime of the donor is: $\tau=k^{-1}=$ $\left(k_{0}+k_{\mathrm{T}}\right)^{-1}$. By using $k_{\mathrm{T}}=k-k_{0}$, Eq. (11) can be rewritten as

$$
k=k_{0}+(\pi / 2) k_{0} R_{0}^{6} r_{\mathrm{a}}^{-4} \rho_{\mathrm{A}} \text {. }
$$


By plotting the decay constant of excited state of the donor, $k$, vs. the acceptor concentration, $\rho_{\mathrm{A}}$, the slope, the second-order rate constant named $k_{2}$, is obtained. Since $R_{0}$ and the acceptor concentration can be determined, for the system where the energy transfer process is dominated by electric dipole-electric dipole transition mechanism, Eq. (9) or Eq. (10) provides a sensitive means of measuring $r_{\mathrm{a}}$.

\section{Results}

Figure 2 shows the decay constant of the ${ }^{5} D_{4}$ state of the $\mathrm{Tb}(\mathrm{pdc})_{3}^{3-}$ as a function of the concentration of $\mathrm{Nd}(\mathrm{pdc})_{3}^{3-}$ at constant concentrations of CTA and $\mathrm{Tb}(\mathrm{pdc})_{3}^{3-}$, the initial part of the decay constant $-\mathrm{Nd}(\mathrm{pdc})_{3}^{3-}$ concentration curve

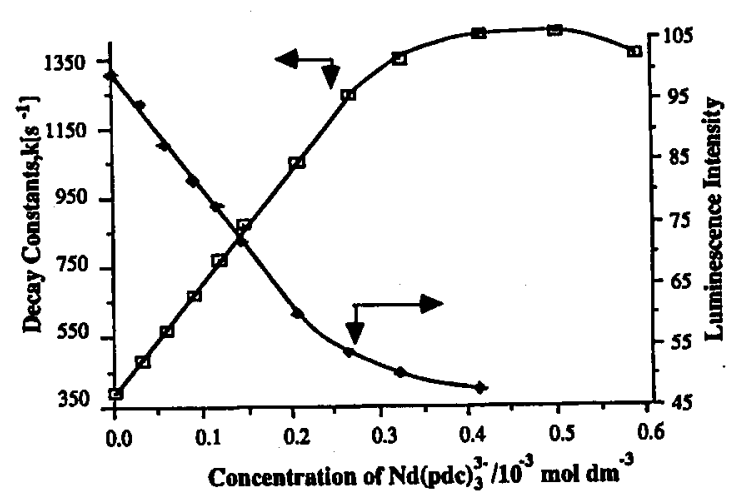

Fig. 2. Luminescent intensity and decay constants, $k$, of $\mathrm{Tb}(\mathrm{pdc})_{3}^{3-}$ as a function of the concentrations of $\mathrm{Nd}(\mathrm{pdc})_{3}^{3-}$ in the solution containing $\left[\mathrm{Tb}(\mathrm{pdc})_{3}^{3-}\right]=$ $1 \times 10^{-4} \mathrm{~mol} \mathrm{dm}^{-3}$ and $[\mathrm{CTAB}]=2.5 \times 10^{-3} \mathrm{~mol} \mathrm{dm}^{-3}$ at $\mathrm{pH}=7.0$.

follows the classical Stern-Volmer quenching law, a linear decrease in the luminescence intensity and a corresponding decrease in the lifetime of donor with the increase in the concentration of the quencher in solution. The quenching constant, $k_{\mathrm{q}}$, evaluated from the slope, however, is not the true quenching constant due to changing of the mean donor-acceptor distance and the two distortion mechanisms of the lanthanide complex anions on the micellar surface as the concentration of $\mathrm{Nd}(\mathrm{pdc})_{3}^{3-}$ is increased. To reduce these variables we performed a series of simplifying measurements in which the ratio of $[\mathrm{CTA}] /\left[\operatorname{Ln}(\mathrm{pdc})_{3}^{3-}\right]\left(\right.$ where $\left[\operatorname{Ln}(\mathrm{pdc})_{3}^{3-}\right]$ refers to the sum of the concentrations of both $\mathrm{Tb}(\mathrm{pdc})_{3}^{3-}$ and $\left.\mathrm{Nd}(\mathrm{pdc})_{3}^{3-}\right)$ is maintained at 4:1 (i.e. maintaining the number of $\operatorname{Ln}(\mathrm{pdc})_{3}^{3-}$ ions on the micellar surface at 20) $[3,4]$, whilst the ratio of $\left[\mathrm{Nd}(\mathrm{pdc})_{3}^{3-}\right]$ and $\left[\mathrm{Tb}(\mathrm{pdc})_{3}^{3-}\right]$ is increased from 0 to 1.5. The distortion of the complex ions and the ratio of bulk solution to surface associated complex ions are therefore constant, the behaviour indicated in Fig. 2 is then due to the change in the number of $\mathrm{Nd}(\mathrm{pdc})_{3}^{3-}$ ions which are nearest neighbours to any particular $\mathrm{Tb}(\mathrm{pdc})_{3}^{3-}$ ion on the micellar surface. The striking feature of Fig. 3 is that the curve becomes strongly non-linear for the 

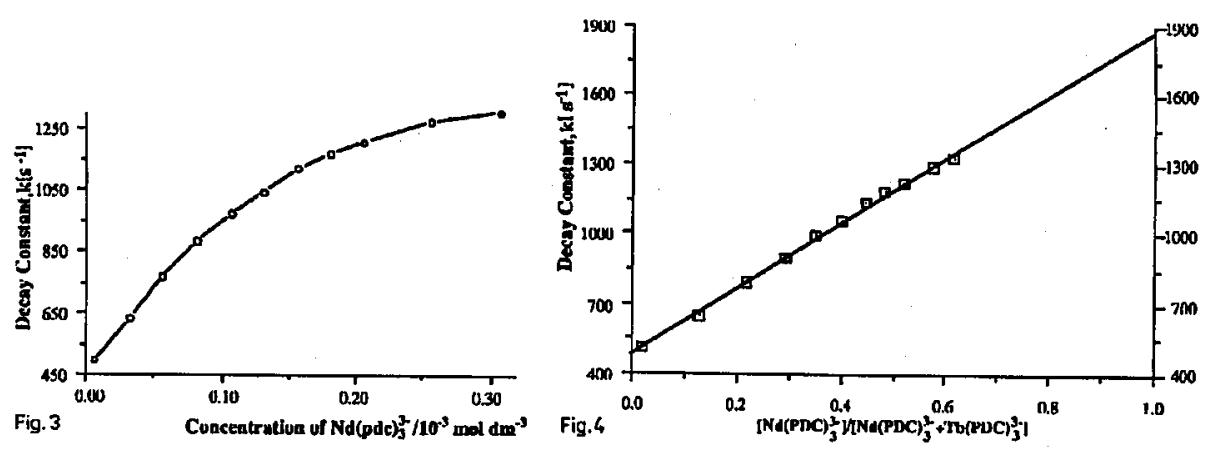

Fig. 3. The decay constant of the ${ }^{5} D_{4}$ state of the $\mathrm{Tb}(\mathrm{pdc})_{3}^{3-}\left(2 \times 10^{-4} \mathrm{~mol} \mathrm{dm}^{-3}\right)$ as a function of the concentration of $\mathrm{Nd}(\mathrm{pdc})_{3}^{3-}$ with the ratio of $\operatorname{Ln}(\mathrm{pdc})_{3}^{3-}$ : CTA held constant at 4:1.

Fig. 4. The decay constant of the ${ }^{5} D_{4}$ state of the $\mathrm{Tb}(\mathrm{pdc})_{3}^{3-}\left(2 \times 10^{-4} \mathrm{~mol} \mathrm{dm}^{-3}\right)$ as a function of the molar ratio of $\left.\left[\mathrm{Nd}(\mathrm{pdc})_{3}^{3-}\right]:[\operatorname{Ln}(\mathrm{pdc}))_{3}^{3-}\right]$ with the CTA : $\left[\operatorname{Ln}(\mathrm{pdc})_{3}^{3-}\right]$ ratio held constant at $1: 4$.

whole range of the $\mathrm{Nd}(\mathrm{pdc})_{3}^{3-}$ concentration. To account for this curvature the microstructure of micelle has to be considered. When acceptor species are present in true solutions, the donor and acceptor species are distributed homogeneously in the whole solution space. In the experimental micelle solutions all the donor and acceptor molecules are associated with the surface of micelle and the total concentration of these species on the micellar surface is constant. However. the quenching constant should relate to the relative concentration of $\mathrm{Nd}(\mathrm{pdc})_{3}^{3-}$ anions on the micellar surface but not of the concentration in bulk solution. Since the micelle concentration in solution is very low, it is assumed that the energy transfer between the different micelles is minimal, i.e. the energy transfer mainly occurs in the same micelle. Re-drawing Fig. 3 by plotting the ${ }^{5} D_{4}$ decay constant of $\mathrm{Tb}(\mathrm{pdc})_{3}^{3-}$ against the molar ratio of $\left[\mathrm{Nd}(\mathrm{pdc})_{3}^{3-}\right] /\left[\mathrm{Tb}(\mathrm{pdc})_{3}^{3-}+\mathrm{Nd}(\mathrm{pdc})_{3}^{3-}\right]$ gives the expected linear result (see Fig. 4).

By extrapolating molar ratio to 1 where each excited $\mathrm{Tb}(\mathrm{pdc})_{3}^{3-}$ ion is assumed to be quenched by all other $\mathrm{Nd}(\mathrm{pdc})_{3}^{3-}$ ions binding on the surface of the same micelle, a limit of the decay constant of $\mathrm{Tb}(\mathrm{pdc})_{3}^{3-}$ (i.e. the limit of quenching) is given by about $1889 \mathrm{~s}^{-1}$ corresponding to an energy transfer efficiency of about $75 \%$. Since the micellar radius is of the order of $2.1 \mathrm{~nm}$ and the maximum number of $\operatorname{Ln}(\mathrm{pdc})_{3}^{3-}$ ion that associated with the surface of a micelle is about 20 , the average distance between metal ions in $\operatorname{Ln}(\mathrm{pdc})_{3}^{3-}$ anions on the micellar surface is about $1.7 \mathrm{~nm}$. The second-order (surface) rate constant, $k_{2}$, is then evaluated as $3863 \mathrm{~s}^{-1} \mathrm{~nm}^{2}$ per $\mathrm{Nd}(\mathrm{pdc})_{3}^{3-}$ ion from Eq. (10) by plotting the decay constant of excited state of $\mathrm{Tb}(\mathrm{pdc})_{3}^{3-}$ against the density of acceptor on the micellar surface. We can now estimate the distance of closest approach between the donor and acceptor species $r_{\mathrm{a}}$.

The critical distance for $50 \%$ energy transfer, $R_{0}$, in Eq. (12) is given by (in 
units of $\mathrm{m}$ )

$$
R_{0}=3.096 \times 10^{-6}\left(k^{2 / 3} \eta^{-4} J \phi\right)^{1 / 6}
$$

where $k^{2 / 3}$ is the orientation factor, $\eta$ is the refractive index of the solvent, $J$ is the spectral overlap integral of the emission and absorption spectra of the donor and acceptor (in units of $\mathrm{m}^{6} \mathrm{~mol}^{-1}$ ), and $\phi$ is the quantum yield of the donor in the absence of acceptor.

The orientation factor, $k^{2 / 3}$, in the usual three-dimensional energy transfer may be taken as $2 / 3$, since metal ions can be treated as isotropic emitters and absorbers. But in two dimensional energy transfer, it increases to 0.847 . Using 1.33 for the refractive index of water, taking the quantum yield for the $\mathrm{Tb}(\mathrm{pdc})_{3}^{3-}$ anion as 0.92 [3], the spectral overlap integral, $J$, for the $\mathrm{Tb}(\mathrm{pdc})_{3}^{3-} / \mathrm{Nd}(\mathrm{pdc})_{3}^{3-}$ pair is about $21.5 \times 10^{-27} \mathrm{~m}^{6} \mathrm{~mol}^{-1}$ which corresponds to a critical distance for $50 \%$ probability of energy transfer by the electric dipole-electric dipole mechanism of $1.3 \mathrm{~nm}\left(R_{0}\right)$.

The mean actual distance between the donor and the acceptor is therefore calculated as $\approx 0.98 \mathrm{~nm}$. This upper limit figure is quite close to the shortest distance of inter-lanthanide separation in crystal of $0.89 \mathrm{~nm}$ [7]. Since the micellar system is highly dynamic rather than static and the $\operatorname{Ln}(\mathrm{pdc})_{3}^{3-}$ ions diffuse rapidly on the micellar surface, the inverse sixth power dependence of the energy transfer rate will ensure however that the occasional close approaches of the donor and the acceptor will make a significant contribution to the energy transfer rate, i.e. heavily weighted the calculated mean actual distance even though the average separation of $\operatorname{Ln}(\mathrm{pdc})_{3}^{3-}$ ions on the micellar surface is about $1.7 \mathrm{~nm}$.

\section{Photophysics of $\mathrm{Tb}(\mathrm{pdc})_{3}^{3-}$ on the surface of phenanthrene containing CTA micelles}

'The excitation spectrum of $\mathrm{Tb}(\mathrm{pdc})_{3}^{3-}$ in aqueous solution shows a medium strength broad band at $31250 \mathrm{~cm}^{-1}$ in addition to sharp $f-f$ transitions due to an $f \rightarrow d$ transition (Fig. 5a). The ligand triplet states may be involved but the precise assignment is not important in this work. Phenanthrene may be readily introduced into the CTA micelle. The absorption spectra of these solutions in the presence of $\mathrm{Tb}(\mathrm{pdc})_{3}^{3-}$ consisted of a simple superposition of the absorption spectra of $\mathrm{Tb}(\mathrm{pdc})_{3}^{3-}$ and micellar phenanthrene. Excitation between 280 and $360 \mathrm{~nm}$ using a broad band continuous arc source excites both emission from the ${ }^{5} D_{4}$ and the phenanthrene fluorescence. The relative intensities of these emissions are excitation wavelength dependent mirroring the different absorption spectra of the two chromophores. In the absence of oxygen, emission from $\mathrm{Tb}(\mathrm{pdc})_{3}^{3-}$ is also excited by absorption into the vibrational levels of the phenanthrene $S_{1}$ state presumably as a result of energy transfer from the organic triplet, since the transfer is largely quenched by the admission of oxygen.

Pulsed excitation at $347 \mathrm{~nm}$ strongly excites emission from the ${ }^{5} \mathrm{D}_{\mathbf{4}}$ state of $\mathrm{Tb}(\mathbf{p d c})_{3}^{3-}$ but the emission decay curves give no evidence for a slow rise time. In marked contrast to those observations the intensity of the emission initially decays more rapidly during a time period comparable to the triplet lifetime (Fig. 6a, b). 


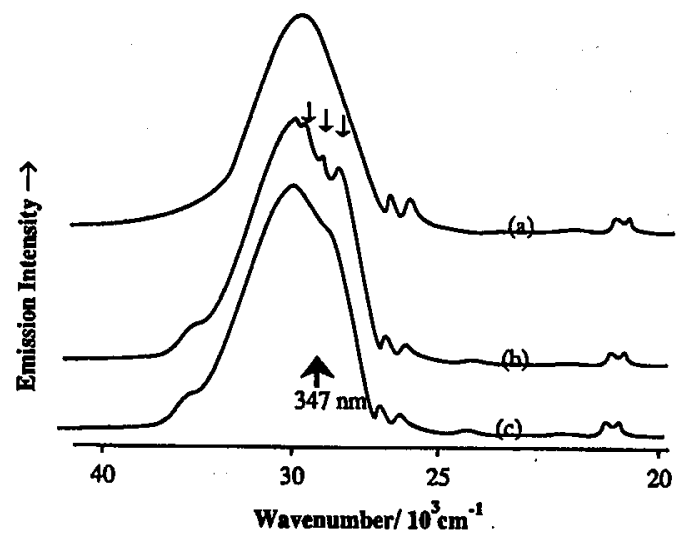

Fig. 5. (a) Low resolution excitation spectrum of a solution containing $8.5 \times$ $10^{-3} \mathrm{~mol} \mathrm{dm}^{-3} \mathrm{~Tb}(\mathrm{pdc})_{3}^{3-}$ and $2.9 \times 10^{-3} \mathrm{~mol} \mathrm{dm}^{-3} \mathrm{CTA}$. The terbium emission was monitored at $18420 \mathrm{~cm}^{-1}\left({ }^{5} D_{4}-{ }^{7} F_{5}\right)$. (b) As (a), but with phenanthrene at $3.3 \times 10^{-4} \mathrm{~mol} \mathrm{dm}^{-3}$ in the absence of oxygen. Note the additional excitation bands due to phenanthrene. (c) As (b) under the same conditions but in the presence of oxygen.

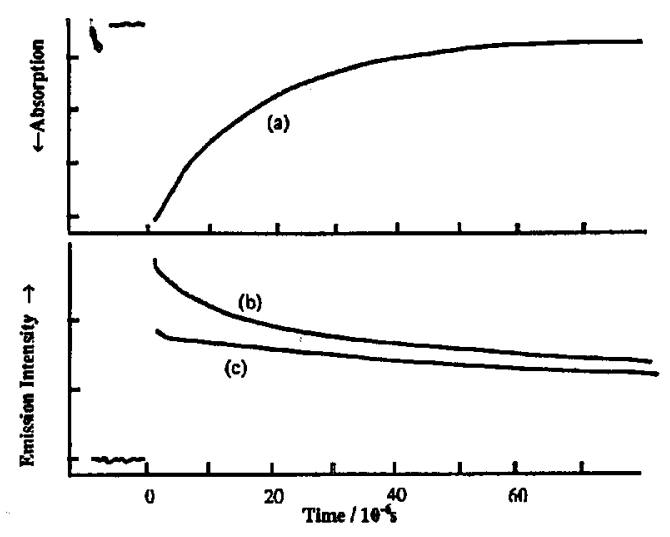

Fig. 6. Kinetic traces for a de-oxygenated solution containing $1.21 \times 10^{-2} \mathrm{~mol} \mathrm{dm}^{-3}$ $\mathrm{Tb}(\mathrm{pdc})_{3}^{3-} 2.9 \times 10^{-2} \mathrm{~mol} \mathrm{dm}{ }^{-3} \mathrm{CTA}$, and $3.2 \times 10^{-4} \mathrm{~mol} \mathrm{dm}^{-3}$ phenanthrene following $28800 \mathrm{~cm}^{-1}$ pulsed laser excitation. (a) Phenanthrene $T_{1} \rightarrow T_{2}$ transient absorption at $20400 \mathrm{~cm}^{-1}$. (b) $\mathrm{Tb}(\mathrm{pdc})_{3}^{3-}-{ }^{5} D_{4} \rightarrow{ }^{7} F_{5}$ luminescence decay monitored at $18420 \mathrm{~cm}^{-1}$. (c) As (b) following the admission of oxygen.

This process is superimposed upon the normal millisecond decay of the ${ }^{5} D_{4}$ state of $\mathrm{Tb}(\mathrm{pdc})_{3}^{3-}$ absorbed onto CTA micelles and is not observed in the absence of phenanthrene. Figure $6 \mathrm{c}$ shows that the admission of oxygen largely removes this additional process thus demonstrating the involvement of the aromatic triplet state in this initial relaxation of the ${ }^{5} D_{4}$ state of $\mathrm{Tb}(\mathrm{pdc})_{3}^{3-}$. We note that $28800 \mathrm{~cm}^{-1}$ 
radiation excites both the phenanthrene $S_{1}$ state and an electron transfer transition of the $\mathrm{Tb}(\mathrm{pdc})_{3}^{3-}$. The former rapidly populates the phenanthrene $T_{1}$ state and the latter the ${ }^{5} D_{4}$ state of $\mathrm{Tb}(\mathrm{pdc})_{3}^{3-}$. The wave number of the ${ }^{5} D_{4} \rightarrow{ }^{7} F_{6}$ transition $\left(20400 \mathrm{~cm}^{-1}\right)$ exactly matches the wavenumber of the $T_{1} \rightarrow T_{2}$ transition maximum.

We propose therefore that the initial rapid decay of the ${ }^{5} D_{4}$ metal localised state is due to resonant energy transfer to the triplet phenanthrene molecules thereby exciting the latter to the $T_{1}$ state.

Thus under intense pulsed excitation conditions the appreciable probability that the same micelle will have a phenanthrene molecule in its triplet state and the $\mathrm{Tb}(\mathrm{pdc})_{3}^{3-}$ in its ${ }^{5} D_{4}$ state allows the possibility of energy transfer from the terbium to the phenanthrene. Under continuous excitation from an arc-lamp/monochromator source the likelihood of such a doubly excited micellar assembly is vanishingly small. This mechanism rationalises all the observations. To explore the necessity of micellar organization we repeated these measurements using the $\mathrm{Tb}\left(\mathrm{OH}_{2}\right)_{9}^{3+}$ ion instead of $\mathrm{Tb}(\mathrm{pdc})_{3}^{3-}$. The former would not be strongly associated with the positively charged CTA micellar surface. The decay curves for emission from the terbium ion and the transient $T_{1} \rightarrow T_{2}$ absorption curves for the phenanthrene solubilisate, both in the presence and absence of oxygen give no evidence for energy transfer. In each case single exponential curves characterised by rate constants equal to those obtained from solutions containing the separate components were obtained.

We thank the British Council for the award of a Technical Cooperation Fellowship to W.D. and the Research Committee of Birkbeck College for financial support. We thank Mr. Phil O'Grady for his continued expert technical assistance.

\section{References}

[1] W. Dong, C.D. Flint, in: Excited State of Transition Elements, Eds. W. Stręk, W. Ryba-Romanowski, B. Jeżowska-Trzebiatowska, J. Legendziewicz, World Scientific, Singapore 1991, p. 327.

[2] W. Dong, C.D. Flint, J. Chem. Soc. Chem. Commun., 1439 (1991).

[3] W. Dong, C.D. Flint, J. Chem. Soc. Faraday Trans. 88, 705 (1992).

[4] W. Dong, C.D. Flint, J. Chem. Soc. Farady Trans. 88, 2661 (1992).

[5] J.R. Darwent, C.D. Flint, N.W. Sharpe, J. Chem. Soc. Chem. Commun. , 747 (1988).

[6] M. Almgren, F. Grieser, J.K. Thomas, J. Am. Chem. Soc. 101, 2021 (1979).

[7] J. Albertsson, Acta Chem. Scand. 26, 1023 (1972). 